\title{
Editors' Note: ISAAC 2009 Special Section
}

Algorithmica gratefully acknowledges the editorial work of the following scholar:

- Ding-Zhu Du, University of Texas, Dallas

on this special section devoted to the 20th International Symposium on Algorithms and Computation.

The papers in this section include:

- "Exact Algorithms for the Bottleneck Steiner Tree Problem" by Sang Won Bae

- "Editing Graphs into Disjoint Unions of Dense Clusters" by Johannes Uhlmann

- "Augmenting the edge connectivity of planar straight line graphs to three" by Csaba D. Toth

- "Tighter Approximation Bounds for Minimum CDS in Wireless Ad Hoc Networks" by Minming Li

- "Extending Steinitz's Theorem to Upward Star-shaped Polyhedra and Spherical Polyhedra" by Seok-Hee Hong

- "An Improved Approximation Algorithm for the Traveling Tournament Problem" by Shinji Imahori

- "Another Sub-Exponential Algorithm for the Simple Stochastic Game" by Decheng Dai 